\title{
Reingeniería para la competitividad de una empresa de la construcción
}

\author{
Brígida Ferrer de Esis * \\ Rixla Villalobos de Weffer** \\ Eudio Omar Barboza F. ***
}

\section{Resumen}

El objetivo de este trabajo es el rediseño de una empresa utilizando la técnica de reingeniería ( $\mathrm{Al})$. Se parte de la premisa que el entorno global exige hoy de las organizaciones una capacidad nunca antes requerida de aprendizaje, cambio e innovación para enfrentar el reto de ser competitivos. La empresa objeto de investigación (PROINCI C.A.) asimiló la idea de éxito asociada a estas capacidades. Optó entonces por rediseñar confrontando los actores principales de cada proceso básico del negocio, con el proceso mismo partiendo del enfoque propuesto por Hammer y Champy. Como resultado se obtuvo una empresa redimensionada, con personal, valores, oficios, estructura y valores integrados y funcionando como un sistema. Se concluye que con la aplicación de esta técnica es posible crear en las organizaciones la cualidad de la competitividad.

Palabras claves: Reingenieria, Competitividad, Aprendizaje Empresarial, Innovación, Cambio Organizacional.

$\overline{\text { Recibido: 22-10-96 }}$ - Aceptado: 10-12-96

- Lic. en Educación. Profesora Titular de la Universidad del Zulia. Venezuela M.Sc en Planificación y Gerencia de Ciencia y Tecnología. Facultad de Ciencias.

** Ingeniero Quimico. Asesora Gerencial de PROINCI. C.A. M.Sc. en Planificación y Gerencia de Ciencia y Tecnología.

*** Ingeniero Civil. Director - Gerente de la Empresa PROINCI C.A. M.Sc en Ingenieria Civil. Especialista en Administración de Empresas. C.C. Delicias Norte. Torre Empresarial. Piso 1. Maracaibo, Venezuela. 


\section{The Re-engineering in the competitiveness of an constructor company.}

\section{Abstract}

The purpose of this paper is to redesign a company using the RI re-engineering technique. The process is based on the assumption that the external environment today demands of organizations an ever-higher capacity for learning, change and innovation in order to contront the competetive challenge. The company under study (PROINCA C.,A.) assimilated the idea of associating success with these capabilities. The company decided to confront the principal actors in each basic process of the business with the total process itself, using tha analysis proposed by Hammer and Champy. As a result, the company was redimensioned, integrating personnel, values, tasks and structures in one functioning system. It was concluded that by applying this technique it is possible to increase competitivity in organizations.

Key words: Re-engineering, Competitiveness, Learning, Innovation, Organizational Change

\section{Introducción}

El entorno global exige hoy de las organizaciones una capacidad nunca antes requerida de aprendizaje, cambio e innovación para enfrentar el reto de ser competitivas y eficientes en todos los aspectos de la vida empresarial; las pequeñas y medianas empresas no escapan a esta tesis. La empresa Proyectos, Inspecciones y Construcciones de Ingeniería, PROINCI C.A., empresa privada de mediano tamaño del sector de la construcción, se dedica básicamente al diseño de proyectos de ingenieria, inspecciones, gerencia y construcción de obras. Esta empresa ha recibido el impacto negativo de la crisis económica que la ha colocado al borde de la quiebra; la crisis económica venezolana significó para el sector de la construcción un decrecimiento que osciló entre un 20 a un $40 \%$ en 1994 . La direc- ción de PROINCl asimiló la idea de éxito asociada a la innovación y al desarrollo de capacidades tecnológicas y entendió que es un camino adecuado para enfrentar sólidamente las amenazas del entorno.

Se optó entonces por rediseñar, inventar nuevas maneras de hacer las cosas y buscar nuevas técnicas para gerenciar el negocio. A partir de este planteamiento se adoptó la técnica de reingeniería ya que es un vía probada para organizar el trabajo de otra manera atendiendo las exigencias del mercado, al cliente como prioridad y al potencial de las nuevas tecnologias que han surgido para el área de ingeniería. Para rediseñar se revisaron los procesos básicos del negocio a la luz de la técnica de reingenieria, es decir, se identificaron los procesos de PROINCIC.A., se diagramaron tal y como se realizaban, se seleccionaron 
los que requerían reingenieria (Ri), se organizaron los equipos responsables de las tareas, se entendieron los procesos en términos generales $y$, por último, se rediseñaron los procesos seleccionados.

Como resultado se obtuvo una empresa redimensionada, con personal, tareas, estructura, administración y valores integrados y funcionando como un sistema; las tareas son multidimensionales, el personal organizado en equipos se evalúa y se paga por sistemas administrativos apropiados lo cual incide en los valores y actitudes de los empleados. Además de estos resultados, se adecuó el tamaño de la empresa a las demandas del entorno, se racionalizaron los costos fijos, y los costos variables se ajustaron de acuerdo a los requerimientos de la empresa. Se considera que PROINCI C.A. marcha hoy a un ritmo adecuado a su entorno y cuenta, como elemento de competitividad, con una Gerencia de Investigación y Desarrollo la cual se consolidó como resultado de la aplicación de la reingenieria.

\section{Aspectos Conceptuales}

La competitividad es un concepto fundamental en las empresas que persiguen como objetivo central mantener y aumentar su participación en el mercado. Para hacerlo es necesario prepararse puesto que la competitividad no es un don natural, es una capacidad que solamente se adquiere con esfuerzo y dedicación. $\mathrm{Si}$ la empresa no es competitiva no puede sobrevivir, menos aún progresar en un ambiente signado por cambios rotundos como es el ambiente de los negocios.
Actualmente las empresas están revisando la manera como hacen sus operaciones para librarse de anticuados métodos de manejar el negocio, están en la búsqueda de un cambio rápido y eficaz. En este escenario surge la reingeniería, técnica diseñada para mejorar la operación de las empresas en aras de un aumento de la calidad y ayudar a las organizaciones a mantenerse en el mercado. La reingeniería es un enfoque reciente, innovador y probado con éxito en diversas organizaciones conocidas en el plano internacional, tales como: IBM Credit, Ford Motors, Kodak, U.S. Sprint, СTB Macmillan/McGraw-Hill, New England Telephone. Se conocen otros casos de éxito a nivel nacional, pero no se ha publicado la información escrita al respecto.

El objetivo central de la reingeniería es el rediseño de los procesos básicos del negocio. Se ha utilizado el término "reingeniería" aún cuando este proceso se puede calificar de varias maneras: transformación, reposicionamiento, reestructuración. Independientemente del nombre que se use, el objetivo final es la búsqueda de la competitividad entendida como la capacidad que tiene una empresa para competir exitosamente, es decir, producir bienes y servicios a bajos costos, excelente calidad y satisfacer las expectativas de sus clientes de mejor manera que sus competidores.

Varios autores han definido el término reingeniería:

Michael Hammer y James Champy (1994:8), creadores del término, proponen entender la reingeniería como "la revisión fundamental y el rediseño radical de procesos para alcanzar mejoras es- 
pectaculares en medidas criticas y contemporáneas de rendimiento, tales como costos, calidad, servicio y rapidez"

Daniel Morris y Joel Brandon (1994: 10)se expresan de esta manera: " Para evitar la proliferación de definiciones de reingeniería, es mejor limitar el uso del término al rediseño de procesos de trabajo de negocios y a la implementación de nuevos diseños. Sin embargo, debe emplearse otro término para abarcar el alcance de esta definición y analizar las otras actividades necesarias, encaminadas a hacer que el trabajo de reingeniería produzca su principal beneficio, la ventaja competitiva. Este término es posicionamiento o reposicionamiento y está dirigido a una visión de más alto nivel y al conjunto de intereses y aplicaciones de la reingenieria para implementar sus directrices".

Para estos autores la reingeniería es un enfoque diseñado para planear y controlar los cambios. Se trata de un rediseño de los procesos de negocio partiendo de un análisis de la posición de la organización para determinar las áreas claves de aplicación y el conjunto de metas que se esperan cumplir, además de preparar el ambiente favorable y de confianza para iniciar los cambios. La propuesta metodológica por ellos presentada se distingue por ser un método de "Reingeniería Dinámica en los Negocios" y proporciona un enfoque sistemático y uniforme para rediseñar los procesos de negocio.

Según Raymond L. Manganelli y Mark M. Klein, (1995:8) la reingeniería es "el rediseño rápido y radical de los procesos estratégicos de valor agregado-
- y de los sistemas, las políticas y las estructuras organizacionales que los sustentan-- para optimizar los flujos del trabajo y la productividad de una organización."

Esta definición establece una diferencia entre los tipos de procesos, y mediante la reingeniería sólo se modificarán aquellos que para la organización son estratégicos y de valor agregado. Los estratégicos son los mas importantes e indispensables en razón de las metas, el posicionamiento y la estrategia de una empresa; los procesos que agregan valor a una organización son los indispensables para satisfacer deseos y necesidades del cliente y por los cuales éste paga. La metodología propuesta por estos autores tiene como característica particular el rediseño rápido y radical.

Dado que el enfoque propuesto por Hammer y Champy es el utilizado para los propósitos de la investigación llevada a cabo en la empresa objeto de estudio, se hará un corto análisis de sus componentes fundamentales.

Cuatro palabras claves integran el concepto propuesto por estos autores: fundamental, radical, espectacular y procesos. La primera palabra clave es fundamental, ella implica preguntarse ¿Por qué la empresa hace lo que está haciendo? ¿Por qué se hace de esa forma?, es decir, preguntarse el qué y el cómo. Estas interrogantes involucran el abandono a los supuestos que la mayoría de los procesos arraigan en las empresas. Según este concepto de reingenieria es necesario determinar primero qué debe hacer una compañía y luego cómo debe hacérlo $y$ eso es lo fundamental del proceso. 
La segunda palabra clave es radical. Significa que los cambios que se buscan no deben ser superficiales, por lo contrario, se debe descartar todas las estructuras y procedimientos actuales e inventar nuevas maneras de realizar el trabajo.

La tercera palabra clave es espectacular. Implica la búsqueda de saltos gigantescos en rendimiento.

La cuarta palabra clave en la definición de Hammer y Champy es procesos, entendida como un conjunto de actividades que recibe insumos y crea un producto de valor para el cliente. Por lo general, las organizaciones fragmentan estos procesos entre los departamentos funcionales que trabajan como estancos aislados; en reingeniería se trabaja en función de los procesos básicos de los negocios los cuales deben ser identificados, entendidos y rediseñados centrando la atención en la búsqueda de la ventaja competitiva de la organización.

A los efectos de la investigación se utilizó el concepto $y$ el enfoque general propuesto por Hammer y Champy para conducir un proyecto de reingeniería en una organización. Se siguieron las reglas generales para iniciar, dirigir y llevar a buen término el proceso que se aspiraba, comenzando con la revisión de la misión y la formulación de un argumento pro-acción. Se continuó con las etapas que estos autores proponen; a juicio de los investigadores responsables de impulsar el rediseño de los procesos en la organización estudiada, las etapas sugeridas permiten, por su amplitud, la libertad de utilizar el ingenio y ajustarlas al caso particular objeto de investigación. En este orden de ideas se instrumentaron las etapas de : 1.Preparación para la $\mathrm{Ri}$, para persuadir a los empleados de la necesidad de rediseñar la compañía, se busca estimularlos para que acojan, o al menos no obstaculicen, la perspectiva de un cambio; 2. identificación de los procesos; 3. organización del equipo; 4. entender el quế y el porqué de los procesos seleccionados $y$, por último, 5. rediseño. La sustentación teórica de cada una de estas etapas se expone simultáneamente con la metodología seguida en su aplicación a objeto de facilitar la comprensión del caso que nos ocupa.

\section{Caracterización de la Empresa PROINCI C.A.}

La empresa PROINCI C.A es una empresa consultora de Ingeniería y Arquitectura fundada en 1981. Cuenta con ingenieros, arquitectos y técnicos de diferentes especialidades, con capacidad para realizar proyectos multidisciplinarios y de responsabilidad total en el campo privado, público y de la industria petrolera y petroquímica. Con este personal se cubren las áreas de:

Proyectos integrales en:
Arquitectura
Ingenieria Civil
Ingeniería Mecánica
Ingenieria Eléctrica.

- Inspección de obras.

- Supervisión de obras.

- Construcciones.

- Gerencia de proyectos y obras 
- Promoción de viviendas.

- Asesorias, consultas y estudios técnicos.

Para prestar un servicio integral, PAOINCI C.A ofrece a través de su empresa afiliada GEOTECNIA S.C los servicios especializados de:

- Estudios geotécnicos de suelos y pavimentos.

- Control de calidad de agregados y obras civiles.

- Consultoría en el área de Geotecnia.

Cuenta con un gran prestigio en la región por su participación en proyectos de importancia tales como edificios institucionales para la Universidad del Zulia y otras Universidades de la región, centros comerciales, edificios médico - asistenciales, museos y estructuras espaciales como la realizada para el museo La Clu- dad de Dios. Su Director - Gerente posee estudios de cuarto nivel tanto en su área técnica como en el área gerenclal.

La alta gerencia decidió revisar la empresa e iniciar un proceso de rediseño, ya que la empresa venezolana debe estar preparada para enfrentar las nuevas realidades socio - económicas vigentes en el país que imponen la adaptación de las empresas a un entorno cambiante, cuya característica macro - económica fundamental es la presencia de una recesión económica y una inflación de hasta un $25 \%$ mensual. En el caso de la industria de la construcción se prevé una caida en la demanda de edificaciones entre un $25 \%$ a un $40 \%$ para el período $95-97$, de mantenerse las condiciones económicas actuales.

La estructura organizativa vigente para el momento en que comenzó este proceso de revisión se observa en el grá-

\section{Gráfico №1}

Organigrama original de la Empresa

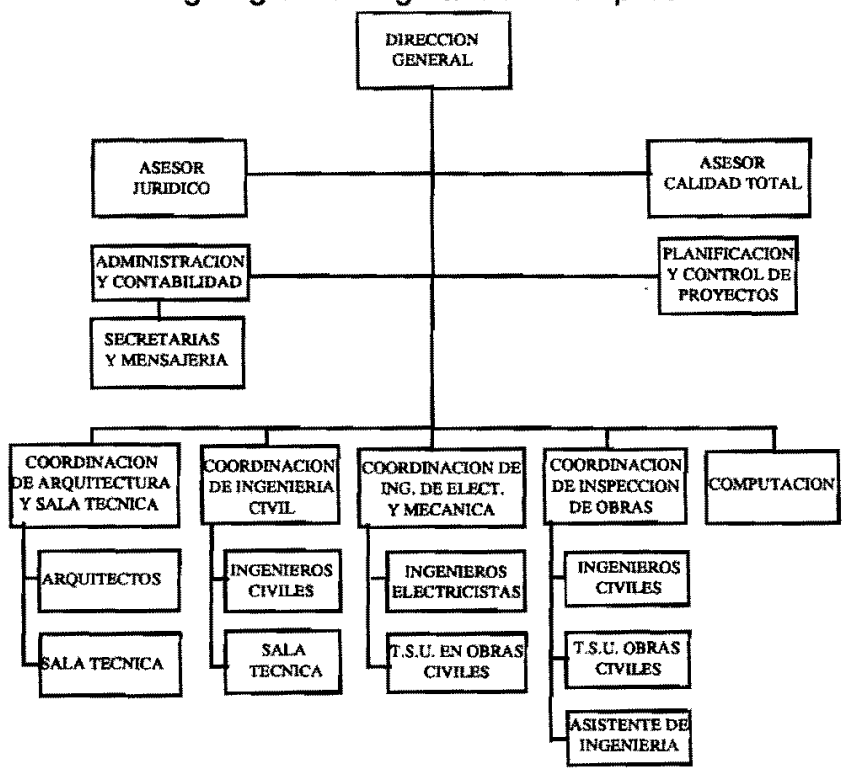


fico № 1 y se caracterizaba por ser funcional, estructurada en base a las especialidades técnicas y burocratizada. En esta estructura el proceso básico del negocio (la realización de proyectos e inspecciones en el área de ingeniería) aparece fragmentado entre las distintas áreas funcionales de la organización..

\section{El proceso de reingeniería}

\section{- Descripción del proceso}

Varias etapas se deben seguir para ejecutar el proceso de reingeniería bajo el enfoque asumido en esta investigación. El proceso comienza con una etapa de preparación que estimula la participación de los empleados en los procesos de cambio, se revisa la misión y se formula el argumento pro-acción. De seguida se identifican los procesos susceptibles de reingenieria utilizando ciertos criterios de selección; se organiza el equipo que realizará la reingeniería en cada uno de los procesos seleccionados, se señala de forma global la manera como se hace tradicionalmente el trabajo, es decir, se entienden los procesos en su concepción general; por último, se rediseña. Cada una de estas etapas se detallan a continuación, se expone su concepción teórica y se describe su aplicación en la empresa objeto de investigación.

\section{Etapa 1: Preparación para ini- ciar el proceso de Ri.}

El propósito de esta etapa es estimular al personal de la organización a reconocer la necesidad impostergable de un cambio indispensable para la supervivencia de la organización. Esta preparación es un requisito insoslayable ya que los empleados que no estén inclinados o dispuestos al cambio pueden ser un fuerte obstáculo para el proceso de rediseño. Una metodología usual para esta primera fase consiste en formular un argumento pro acción; el mismo es un argumento a favor de la acción, como dice Hammer y Champy (1994: 156) "dramáticamente convincente, apoyado en hechos concretos" y que demuestra que si no se hace un camblo radical, la empresa pierde su ventaja competitiva porque realmente está en dificultades. En este caso, el argumento pro-acción fue la poca rentabilidad que exhibia la empresa y la amenaza de su cierre si no se lograba transformar.

Este reconocimiento de la necesidad de un cambio es impulsada bien sea porque las organizaciones sienten "dolor" al ver que, por ejemplo, pierden participación en el mercado o porque sus utilidades son muy bajas o simplemente no hay utilidades; porque las organizaciones sienten "temor" ante la alta incertidumbre de su entorno específico, o bien porque las organizaciones tienen "ambición" y desean, por ejemplo, aumentar la participación en el mercado. Ante cualquiera de estos tres impulsos la organización necesita hacer algo y pronto.(Manganelli $y$ Klein, 1995:56)

En el caso de la empresa objeto de esta investigación el proceso de cambio se inicia ante el "temor" de perder su participación en el mercado de la construcción con el que contaba, dado que venía presentando graves problemas de liquidez ocasionados por unos gastos $\mathrm{f}$ - 
jos operativos altos. Esta situación incidia en las posibilidades de apuntalar las fortalezas de la organización con las cuales se hacia frente a las amenazas del entorno, por ejemplo, las limitaciones económicas afectaban la incorporación de personal calificado para la ejecución de actividades de investigación y desarrollo de nuevas tecnologías aplicables al área de la construcción.

Ante la demostración sincera de los criticos aspectos contables de la empresa los empleados aceptaron el reto de un rediseño en sus procesos de trabajo. La alta gerencia de la empresa conocia ya de otras experiencias de aplicación de reingeniería de procesos con resultados exitosos; las características innovadoras propiciaron la investigación sobre el tema aunado a la lectura y discusión de los textos que circulaban sobre reingeniería. En este ambiente se incorporaron los consultores - investigadores que actuarían como catalizadores del rediseño de los procesos. Estos fueron los pasos iniciales; de las minutas de las reuniones se recogen los siguientes aspectos claves:

El proceso comenzó con una conferencia introductoria en la cual se presentó la filosofía de la reingeniería en base a los siguientes criterios: conceptos; pertinencia, en base al argumento "pro acción" que impulse el cambio; rediscusión sobre la misión y visión de la organización; revisión de las metas organizacionales; explicación formal de la planificación del proceso de reingeniería (Ri) que se llevaría a cabo, destacando el compromiso de la alta gerencia de la empresa.

La etapa de preparación tuvo una duración de aproximadamente tres se- manas, con diez horas semanales de trabajo; en estas sesiones se discutió en profundidad el plan propuesto, la filosafía de la Ri y la necesidad impostergable de hacer algo antes de llegar a límites insalvables. En estas reuniones participó siempre la alta gerencia y se hacían por grupos de empleados.

\section{Etapa 2: \\ Identificar los procesos.}

Cumplida la fase de preparación fundamental, y considerando las asesoras externas que la empresa estaba a tono para comenzar, se dio inicio a la etapa de identificación de los procesos claves, aquellos que estratégicamente agregan valor al resultado final que se entrega al cliente. En esta etapa se trata de identificar qué procesos se deben rediseñar y comprender estos procesos a la luz de las necesidades de los clientes. Por lo general las organizaciones tienen uno o varios procesos fundamentales que deben fluir de forma natural; sin embargo, la organización por si misma los fragmenta entre los departamentos funcionales y cada jefe de departamento debe garantizar una parte del trabajo; nadie por lo tanto es responsable del proceso global.

Se le pidió al personal involucrado en la Ri que graficaran el proceso general que se llevaba a cabo sin tomar en consideración la estructura formal, ni los cargos ni los diferentes departamentos. La clave consistió en responder a la pregunta ¿Qué hace PROINCl? visualizando esta operación desde el primer contacto con el cliente hasta la entrega del producto generado por la compañia. Los profe- 
sionales, con la visión global del proceso, pudieron elaborar tres diagramas con más de doce pasos cada uno y con ligeras variantes en cada caso. Se muestra uno de ellos para ilustrar. (Ver Gráfico No. 2) . Así, se identificaron cuatro procesos comunes:
a) Negociación con el cliente.
b) Ejecución del trabajo solicitado
c) Entrega al cliente del producto final.
d) Servicio de Post-venta.

Tomando en consideración estos procesos, en principio los básicos para el propósito de la empresa y los más generales por permitir englobar sus funciones, se procedió a evaluar la viabilidad de la Ri en cada uno de ellos. Para este propósito se utilizaron los siguientes criterios : a) disfunción, b) importancia, c) factibilidad. (Hammer y Champy, 1994:129-136)

Estos criterios responden a lo siguiente:

a) Disfunción: Implica el reconocimiento de dificultades en los procesos a través de la detección de ciertos síntomas tales como extenso intercambio de información, redundancia de datos, exceso de inventario, control exagerado a través de todo el proceso, retrabajo ocasionado por falta de coordinación, escasa información para ejecutar una parte del proceso, entre otros. Estos son síntomas comunes en muchas empresas que revelan dificultades o problemas serios en alguna parte del proceso.

b) Importancia: La importancia del proceso se mide en función del impacto sobre el cliente, la pregunta clave es ¿Cuál proceso ejerce el mayor impacto sobre la clientela de la empresa? el fin es determinar qué cuestiones le interesan primordialmente al cliente, es decir, costo, calidad, tiempo de entrega, etc. Las respuestas a estas interrogantes dan la pauta para establecer el orden de prioridades para la reingeniería de procesos.

c) Factibilidad: Este tercer criterio implica determinar las probabilidades de éxito del esfuerzo que se realizará. En este sentido se afirma que mientras más grande sea el radio de acción del rediseño de procesos menos probabilidades de éxito tendrá. Igual sucederá si implica un alto costo o si el equipo de reingeniería no está del todo comprometido.

Al lado de estos tres criterios se deben utilizar otros tales como la comparación de la manera como se hacen las cosas en otras compañías exitosas del área o preguntarse si el proceso es o no anticuado. Mientras más argumentos se acumulen a favor del rediseño más probabilidades de éxito se tendrá.

Aplicados estos criterios a los procesos identificados se concluyó que era necesario cambiar los procesos de "negociación con el cliente" y el de "ejecución del trabajo". Por el mayor impacto del segundo sobre el cliente se recomendó iniciar alli el proceso de $\mathrm{Ai}$.

El siguiente paso fue organizar el equipo para cada uno de los procesos seleccionados; así se conformó un equipo para "negociación con el cliente" y otro para "ejecución del trabajo"; sin duda que la prioridad la tenía el proceso denominado "ejecución del trabajo".

\section{Etapa 3: Organizar el equipo.}

El paso siguiente para el rediseño 


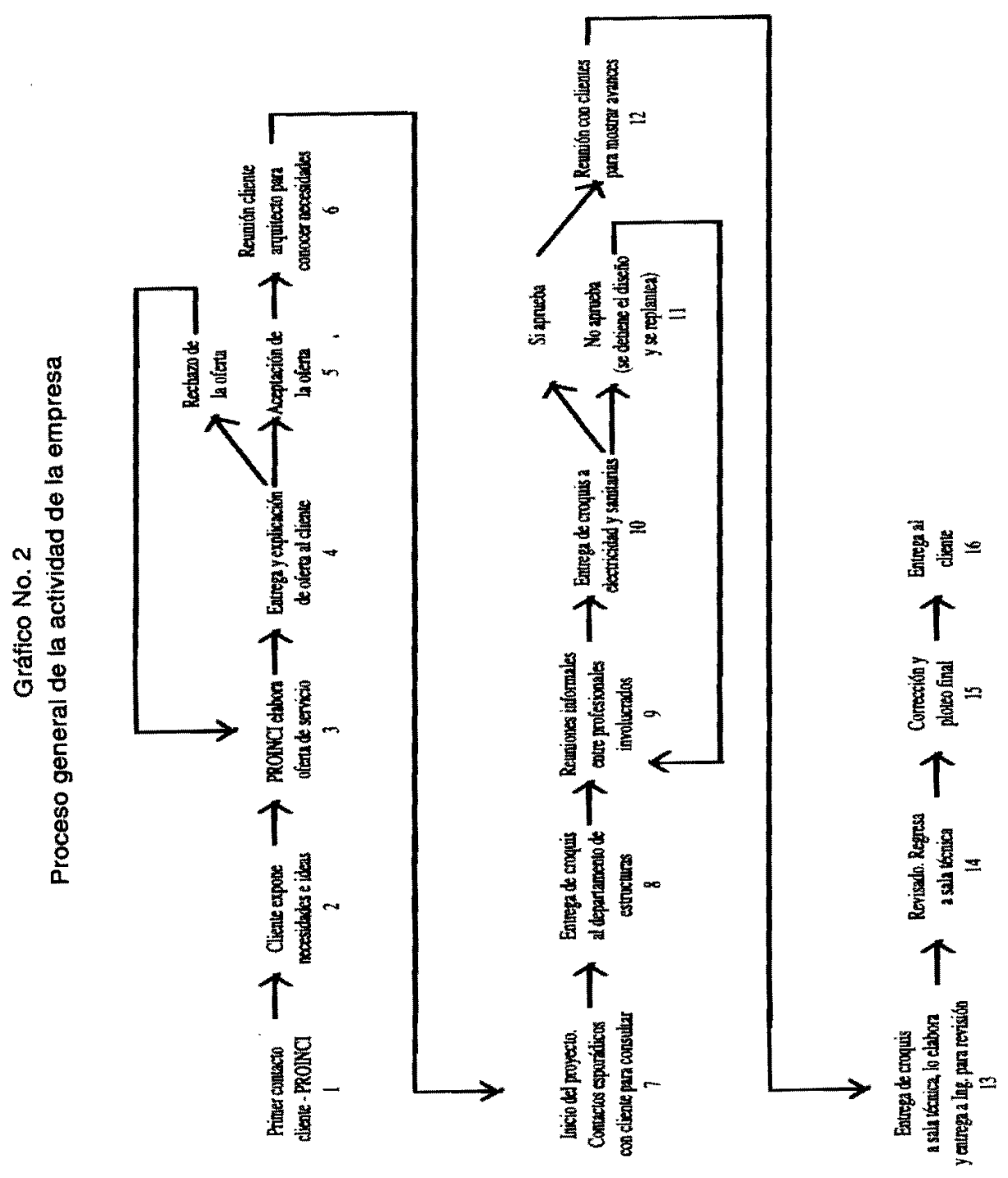


de los procesos es la organización del equipo que realizará la $\mathrm{Ri}$. A tales efectos Hammer y Champy (1994: 108-123) proponen la conformación de un equipo en el que están presentes un líder que autoriza y motiva el esfuerzo total; un dueño del proceso que es la persona responsable de un proceso específico y del esfuerzo de Ri enfocado en él; el equipo de reingeniería dedicado a diagnosticar el proceso, rediseñarlo y supervisar su ejecución; un comité directivo formulador de políticas, responsable del diseño de la estrategia global de la organización y supervisión de su progreso y, un zar de reingeniería responsable de desarrollar técnicas e instrumentos de $\mathrm{Ai}$ y lograr la sinergia necesaria entre los distintos grupos de Ri de la empresa.

En PROINCIC.A estas designaciones no se hicieron siguiendo fielmente lo expuesto por Hammer y Champy, en virtud de que se estima que cada compañía diseña su equipo bajo su propio enfoque adaptado a su naturaleza y tamaño. Por ello se nombró al líder, al dueño del proceso, al equipo y a un grupo de personas, incluidas las asesoras externas, que conforman el comité técnico asesor (CTA).

Un alto ejecutivo, el Director - Gerente, asumió la función de líder del proceso dado su convencimiento de la necesidad de reinventar la empresa; con una visión clara de hacia dónde llegar, fue capaz de contagiar con carácter el entusiasmo hacia todo el personal para lograr el esfuerzo y la sinergia requerida. El proceso fue decretado, asumido y apoyado por el líder.

Los dueños del proceso, responsables de rediseñar por separado los dos procesos seleccionados, fueron nombrados tomando en cuenta el grado de compromiso con la organización, además del prestigio y autoridad frente a la tarea que se va a rediseñar y frente al grupo de trabajo constituido para cada proceso. Además de estos atributos, los dueños de cada proceso deben hacer que el trabajo se ejecute, suministrando no solo energía y entusiasmo sino también recursos y apoyo de cualquier otro miembro de la organización que tenga relación con el proceso. Al mismo tiempo actúan como críticos, protectores y estimuladores del trabajo. Tomando en cuenta estas consideraciones se nombraron dos ingenieros que ocupaban importantes posiciones en la empresa para que cumplieran con este rol.

El equipo de reingeniería asume la carga más fuerte de trabajo al tener que producir las ideas y los planes y llevarlos a cabo; son, como dicen Hammer y Champy, los que en la práctica reinventan el negocio. Cada uno de los dos procesos seleccionados contó con un equipo conformado por siete personas, de ese equipo dos personas participaron en los dos procesos ya que eran las consultoras externas que debian darle seguimiento $y$ poner el toque de visión objetiva desde afuera, colaboraron con el líder, programaron las reuniones, trazaron las estrategias y mantuvieron siempre presente la perspectiva de cambio, es decir, asumieron las tareas propias del zar de reingeniería. Además de estas funciones, las consultoras externas tenían el interés particular de la investigación con fines académicos y contaron con un año libre de docencia para esta investigación. La 
realización de este proyecto es una manifestación clara del apoyo que presta la Universidad del Zulia al sector productivo de la región.

La última designación realizada para el proceso de rediseño fue la del comité directivo o comité técnico administrativo (CTA). Aún cuando puede ser un aspecto opcional, en el caso de la empresa que nos ocupa, es una función permanente dentro de la organización. Se define como el grupo de altos administradores, que junto con los dueños de procesos y el líder proyectan la estrategia global de la Ri de la organización. Es un cuerpo de discusión donde se exponen problemas que escapan a los miembros designados y se buscan soluciones por consenso. En PROINCI esta figura funciona como un órgano de staff que apoya la ejecución de la gerencia; es una fortaleza de la empresa porque alli concurren permanentemente todos los gerentes operacionales, la alta gerencia y los asesores externos; dependiendo de las situaciones que se ventilen se incorporan las personas involucradas, por ejemplo, el administrador, el asesor legal $u$ otros.

Este comité técnico administrativo se encargó de diseñar la estrategia global de Ri de la organización y de darle el seguimiento correspondiente.

Cumplida la conformación de estos equipos y las designaciones a que hubo lugar se dio inicio al siguiente paso: entender el proceso y diagramarlo.

\section{Etapa 4:}

\section{Entender los procesos.}

Consiste substancialmente en en- tender el qué y el porqué del proceso, mas no el cómo ya que esta última pregunta es la esencia del proceso de rediseño, objeto de la siguiente etapa. Para entender el qué y el por qué se debe pensar en función de las necesidades del cliente ya que el objetivo último de la $\mathbf{R i}$ es el rediseño de los procesos para satisfacer las metas y resolver los problemas de los clientes. El equipo de Ri ya conformado requirió revisar aspectos generales del proceso, las preguntas claves que se formularon fueron: ¿Qué es lo que se hace? ¿Por qué se hace? ¿Existen cuellos de botella en los procesos? y responder estas preguntas sin caer en detalles específicos o análisis pormenorizados.

El equipo número 1 conformado para el rediseño del proceso de "ejecución del trabajo" comenzó por entender las diferentes modalidades para el desarrollo de un proyecto:

1.- Cuando el cliente aporta el diseño de arquitectura.

2.- Cuando el cliente requiere que el diseño de arquitectura se realice en la empresa y se parte sólo de las ideas generales que tiene el cliente.

3.- Cuando el cliente tiene el diseño de arquitectura y los cálculos de ingeniería y busca únicamente la gerencia de la obra.

En cualquiera de los casos el proceso que se sigue es creativo y no se encierra en una fórmula única, aún cuando el cliente siempre quiere calidad, bajo costo y cumplimiento de la fecha de entrega. Partiendo de estos pre requisitos del cliente comienza un flujo de relaciones o interacciones entre los distintos entes involucrados para darle satisfac- 
ción a sus necesidades. Aquí se detecta un nudo crítico, los ingenieros y arquitectos no se sitúan en el lugar del cliente, el prestigio ganado por PROINCl en su ámbito geográfico genera en sus empleados un sentimiento de sabiduría y poder que les impedía interpretar las ideas del cliente y terminaban estructurando el proyecto desde la óptica de la empresa, casi que imponiéndole al cliente sus ideas fundamentadas en argumentos técnicos y criterios de calidad y eficiencia; la perspectiva del cliente iba quedando a un lado, no eran capaces de interpretar lo que el cliente quería y lo que realmente necesitaba.

Además de estas consideraciones sobre la importancia de entender el proceso desde la óptica del cliente se esquematizó el proceso en sí de ejecución del trabajo. En general, se describió el proceso como "soluciones de ingenierla" sin entrar en detalles sobre las diferentes modalidades descritas para el desarrollo de un proyecto. Estas soluciones se producen con la participación de un grupo de empleados que asume cada uno una parte del trabajo y lo realiza individualmente, es decir, cada profesional hace su parte y se supone que lo está haciendo bien, de acuerdo a su juicio profesional. En resumen sucedía lo siguiente: los ingenieros inician el proceso y entregan sus borradores a los dibujantes de acuerdo a las primeras ideas expuestas por el cliente y los primeros cálculos. Por lo general se aceptaba que esta primera entrega era una aproximación para confrontar las aspiraciones del cliente con las percepciones de los ingenieros responsables del cálculo; los resultados sólo alcanzaban el
$50 \%$ de lo esperado. Los principales problemas que exhibía el proceso original eran los siguientes:

- Se establecía una "relación perversa" entre los dibujantes y los ingenieros responsables del cálculo: los ingenieros modificaban y devolvian a la mesa de dibujo y viceversa, sin existir una comunicación directa que eliminará el retrabajo.

-El retrabajo consumía recursos materiales físicos, financieros $y$, lo que quizás es más importante, corroe el estímulo de los empleados.

-Por lo general se sabía lo que quería el cliente cuando devolvía el producto final ya terminado, porque no existían etapas intermedias de interacción clienteempresa a través de las cuales el cliente conociera los avances de su proyecto y formulara sus observaciones.

Para el caso del segundo proceso objeto de rediseño la "negociación con el cliente" esta etapa se cumplió cuando el equipo describió cómo era el proceso vigente para ese momento de negociación con el cliente; éste se fundamentaba en tres fases caracterizadas por los siguientes elementos:

Fase 1: Búsqueda en el mercado de oportunidades para PROINCI por parte del Director - Gerente. Estas oportunidades también se presentaban cuando los clientes acudian a la empresa por referencia de otros clientes o por conocimiento del prestigio de la organización. EI Director, hábil para la comunicación y la persuasión, lograba que el cliente contratara su proyecto con la empresa.

Fase 2: En esta fase se recopilaba toda la información para preparar la oferta al cliente. Aquí se presentaba un nudo 
crítico por la fragmentación de la información suministrada por los diferentes profesionales de cada especialidad involucrados en el proyecto; por ejemplo, al momento de establecer el cálculo de las horas - hombre necesarias se manejaba información no ajustada a la situación real en relación al costo y tiempo necesarios para la ejecución de trabajos. En consecuencia, se estipulaban presupuestos muy altos que luego tenian que ser arreglados de acuerdo al mercado. Esto ocasionaba pérdidas de tiempo y retrabajo.

Fase 3: En esta fase se presentaba la oferta definitiva al cliente y se daba la negociación cliente - empresa hasta llegar a acuerdos.

Fase 4: Se firmaba una carta convenio si el cliente era conocido, de lo contrario el abogado elaboraba un documento notariado.

El proceso de negociación con el cliente realizado de esta manera tenía serios inconvenientes entre los cuales se resaltaron:

- La promoción se hacía únicamente a través del Director-Gerente, quien asumía la responsabilidad de promoción limitándola a su entorno de influencia.

- El equipo técnico responsable de la ejecución del proyecto no estaba presente en la negociación con el cliente, por lo tanto se perdia una información de primera mano.

- El Director-Gerente no contaba con la estructura de costo real en el momento de la negociación por la ausencia en la organización de mecanismos de control y aseguramiento de información acerca de estos aspectos.

- Incumplimiento de pagos por par- te de terceros por falta de formalización en la firma de los contratos.

\section{Etapa 5: \\ Rediseñar los procesos.}

Entendidos los dos procesos objeto de rediseño se comenzaron a especificar los términos en que se deben dar los nuevos procesos; cada uno de los miembros de los equipos de Ri fueron aportando ideas que permitieron reconducir tanto el proceso de "ejecución del trabajo" como el de "negociación con el cliente" y disminuir el retrabajo; se examinaron las relaciones entre las distintas etapas que originalmente se cumplian para cada uno de los procesos objeto de rediseño. Se trata en esta etapa de inventar una nueva manera de hacer el trabajo y lograr mayor rendimiento.

En caso del proceso de ejecución del trabajo se comenzó esta etapa con una tormenta de ideas sobre los nudos críticos que exponía el proceso actual y la búsqueda de opciones viables para resolverlos. En este sentido se consideraron varios aspectos claves:

- Diseñar alternativas para disminuir el retrabajo. Ej: automatizar las tareas repetitivas.

- Coordinar las distintas fases que se definan para el nuevo proceso, se analizó la necesidad de nombrar un líder de proyecto, responsable de la coordinación de todas las fases y de la ejecución del trabajo frente al cliente.

- Disponer de una base de datos para preservar la información que se maneja rutinariamente en los proyectos..

- Disponer de herramientas alta- 
mente competitivas; en este sentido se planteó la idea del adiestramiento de todo el personal de ingeniería en el manejo de AutoCAD y Hoja de Cálculo. El AutoCAD es una herramienta que permite realizar toda clase de dibujos técnicos $203 \mathrm{di}$ mensiones: esto incluye entre otros, dibujos relacionados con todas las ramas de la ingeniería: diseños mecánicos, eléctricos, electrónicos, de arquitectura, planos, etc. Las aplicaciones CAD son herramientas muy poderosas. La velocidad y facilidad que caracteriza al dibujo asistido por computadora proporciona una ganancia de tiempo importante, además que permite al usuario disponer en un computador personal de una tecnología sofisticada reservada a sistemas grandes y costosos.

Luego de explorar estas alternativas se comenzó a rediseñar el proceso formulando una serie de cambios para simplificar las tareas, eliminar el retrabajo, reducir el tiempo y los costos de operación y satisfacer de mejor manera al cliente. Se inventó una nueva manera en la cual quedaron eliminadas aquellas actividades que no agregaban valor al producto final, por ejemplo: relación dibujan- te-ingeniero proyectista, ahora el ingeniero dibuja directamente; relación clientegerente general, ahora es cliente-equipo de trabajo. El nuevo proceso de "ejecución del trabajo" comienza con una reunión en la que simultáneamente interactúan el cliente, los ingenieros responsables del cálculo y los dibujantes, es decir, en lugar de una cadena de actividades que comenzaban por entender a medias las necesidades del cliente, seguido por una interpretación sesgada por parte de los responsables del cálculo y, el dibujo aproximado de lo que se quería, se propicia desde el inicio un acercamiento de los distintos actores claves, buscando acortar la cadena y disminuir el tiempo de ejecución (Fase 1). Estas reuniones de equipo, integrado alrededor de un líder, deben continuar periódicamente a lo largo del proceso de planificación y ejecución del trabajo (Fase 2 y Fase 3). Las reuniones donde participa el cliente para exponer su opinión son de suma importancia para el nuevo proceso ya que todos los actores interactúan y reciben información directa y simultáneamente.

El nuevo proceso rediseñado se diagramó de la siguiente manera:

Gráfico № 3

Nuevo proceso de ejecución de proyectos

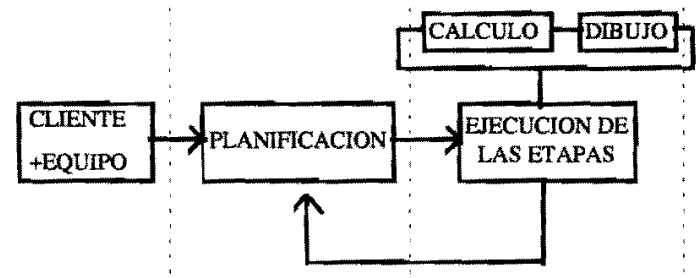

FASE 1

FASE 2

FASE 3 
Para el segundo proceso objeto de rediseño, la "negociación con el cliente", se llevaron a cabo varias reuniones con la finalidad de discutir problemas puntua. les del viejo método; con el mismo enfoque utilizado para el caso anterior, es decir, teniendo como norte la satisfacción del cliente, se organizó una tormenta de ideas a fin de buscar alternativas para el nuevo proceso. Se analizó como muy importante la fase inicial de captación de clientes y el "enamoramiento" necesario para engancharlo con la idea de realizar su proyecto en la empresa analizada. Se consideró substancial que el cliente observara el grado de organización que se posee; en consecuencia, se acordó como paso inicial invitar al cliente a conocer la empresa y mostrarle sus instalaciones.

Esta inducción del cliente debe ser realizada por alguno de los gerentes con más tiempo en la organización y debe ser complementada con trípticos informativos, la carpeta de presentación de la empresa y una información audiovisual sobre las obras más importantes ejecutadas por ella en la región. Este conjunto de actividades se deben instrumentar con clientes particulares $u$ organizaciones interesadas en el servicio que ofrece la empresa; así mismo, se acordó la ejecución de un conjunto de acciones tendientes a promocionar en la región los servicios que se ofrecen y difundir en eventos, congresos, vallas publicitarias y presentaciones en colegios profesionales del área de ingeniería, los logros alcanzados tanto organizacionales como en la ejecución de proyectos y gerencia de obras. La estrategia para el caso de los logros alcanzados en cuanto a investigación en el área de software serán expuestos en el aparte referido a primeros resultados. EI equipo de Ri organizado para el rediseño de este proceso de negociación con el cliente consideró que esta primera fase de inducción es clave (Fase 1).

La reunión simultánea del equipo que va a ejecutar el trabajo, el cliente y el lider del proyecto es el segundo aspecto importante en el nuevo proceso de negociación, con esta fase se trata de detectar con precisión las necesidades del cliente, quien expone sus ideas y es escuchado, $a$ fin de establecer con claridad los elementos indispensables para poder satisfacer tales necesidades. Estos elementos son: horas - hombre, equipos, materiales, tiempo de ejecución, cambio de especificaciones y cualquier otro costo directo o indirecto. La información suministrada por el cliente es recibida directamente por cada uno de los miembros del equipo con la intención de disminuir el margen de error en los cálculos de costos del proyecto. Como resultado de esta reuniones surge la posibilidad de una reestructuración del equipo que ejecutará el trabajo; la idea es que el equipo se conforme con los profesionales necesarios para satisfacer plenamente los requerimientos del cliente (Fase 2).

Cumplida esta fase, el equipo de profesionales se reúne para afinar los costos involucrados, la necesidad de incorporar o no recursos humanos, planificar el trabajo y determinar el tiempo de ejecución (Fase 3). Luego se elabora la oferta con la participación de las oficinas administrativas y legal. Esta oferta se le presenta al cliente, es revisada y negociada tantas veces como sea necesaria a la 
luz del tipo de negociación de "ganar-ganar" (Fase 4), luego se firma el contrato notariado (Fase 5). El diagrama del nuevo proceso de "negociación con el cliente" se observa en el gráfico No. 4.

\section{Primeros Resultados}

\section{- Rediseño de la estructura or- ganizativa.}

Las interdependencias de los nuevos procesos son claras y se conoce con exactitud quienes son los actores involucrados en cada etapa. Esto obligó a una revisión de la estructura organizativa de la empresa concluyendo en una propuesta de organigrama mucho más plana y flexible que el organigrama vigente en PROINCl antes de la Rí. El nuevo organigrama se muestra en el gráfico No. 5
El nuevo organigrama refleja una estructura matricial, válida para empresas de ingeniería, la cual dada su naturaleza, requiere tanto de una alta especialización en las disciplinas afines de la ingeniería civil como de la integración de sus recursos físicos y humanos para generar un producto homogéneo y de alta calidad.

La estructura matricial propuesta presenta en la parte superior de la figura las funciones familiares de Dirección, Administración, Consultoría Jurídica, Consultoria Gerencial y Comité Técnico Administrativo. Como unidades operativas se establecen las Gerencias de Desarrollo e Investigación, Gerencia de Proyectos y Gerencia de Obras, desde alli se derivan los diversos equipos que atienden los proyectos que adelanta la empresa.

\section{Gráfico $\mathbf{N}^{2} 4$}

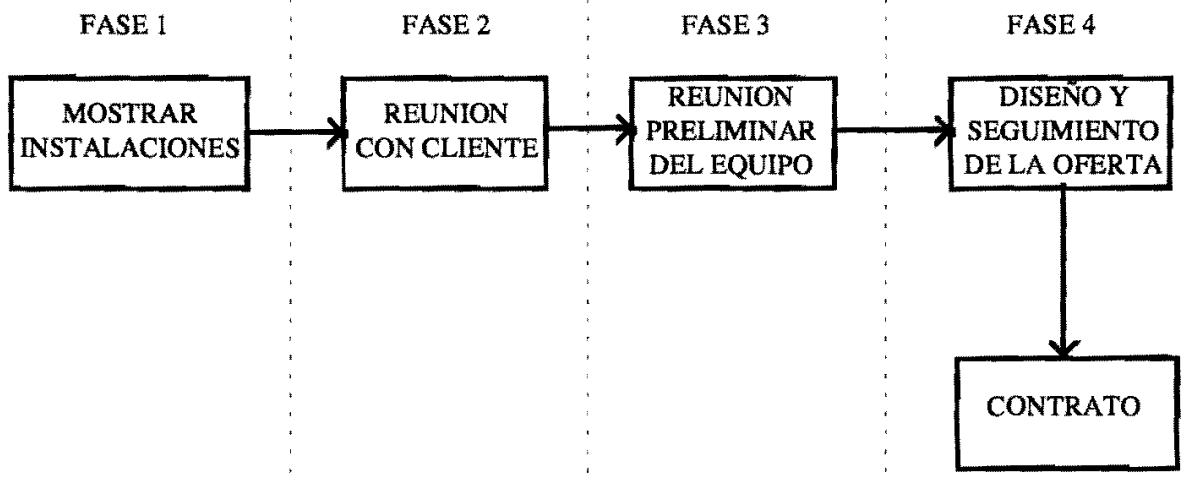

FASE 5 


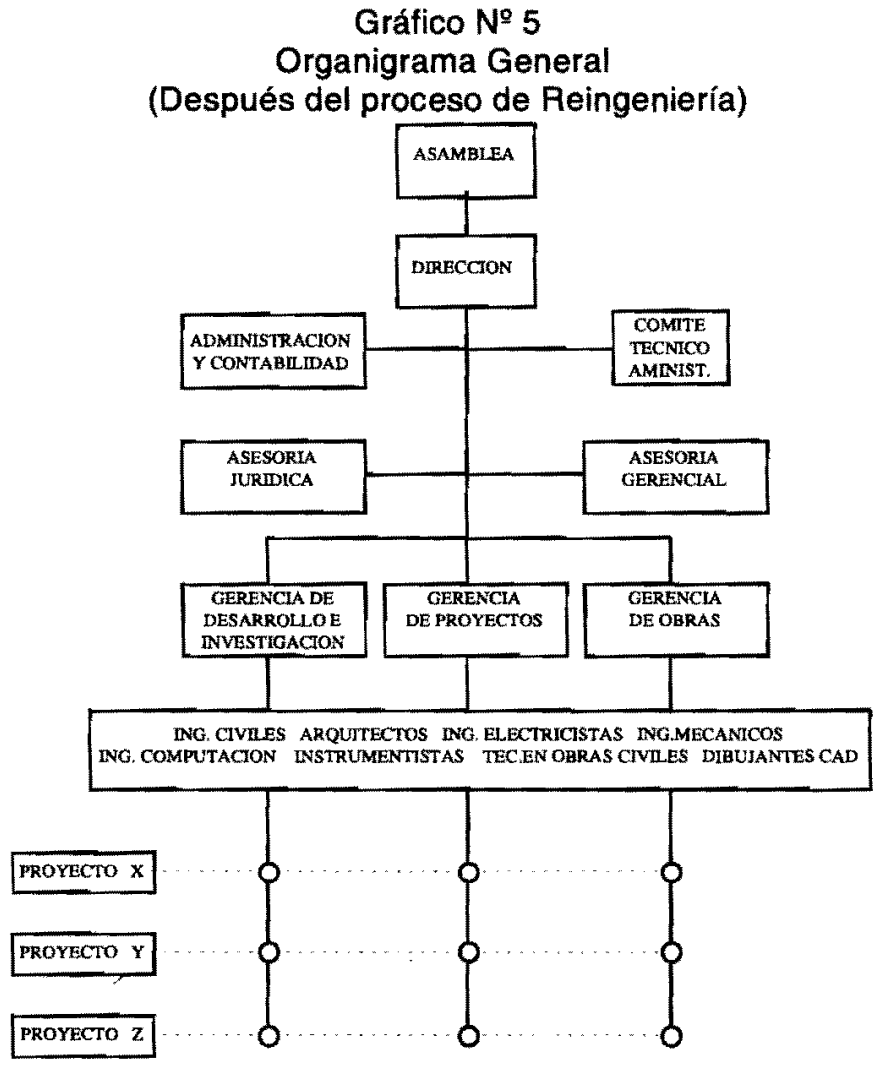

\section{Fijación de políticas de comunicación.}

Se estableció una política de comunicación en la empresa a través de carteleras y reuniones una vez a la semana con todo el personal a fin de darle seguimiento a los procesos rediseñados y resolver cualquier conflicto que se pudiera presentar.

\section{- Nuevo sistema de remuneraciones.}

De igual manera se revisó el siste- ma de remuneraciones. A tal efecto se acordó darle participación a los líderes y a su equipo en los beneficios resultantes por la aplicación de los nuevos procesos. La nueva forma de remuneración consis. te en el pago por honorarios profesionales de acuerdo a la participación en cada uno de los proyectos adelantados. En el caso del personal de I\&D permaneció la relación laboral en su calidad de empleados fijos debido a la necesidad de garantizar la estabilidad en el mediano y largo plazo de este personal especializado y además por la imposibilidad de medir sus resultados en el corto plazo. 


\section{- Creación de la Gerencia de Investigación y Desarrollo.}

Como resultado de la Ri llevada a cabo se creó la Gerencia de Investigación y Desarrollo, a través de la cual se genera tecnología adecuada a las necesidades de la empresa tratando de maximizar el uso del recurso humano el cual debe estar educado para hacer uso efectivo de las herramientas tecnológicas que se están diseñando. La creación del software necesario para ejecutar pasos de los procesos rediseñados en menor tiempo que las empresas de la competencia establece una ventaja competitiva. Justamente se produce lo que se necesita y a la medida de la organización, de ningún modo estos programas de computación se pueden comprar porque simplemente no existen en el mercado.

Ejemplo de estas afirmaciones lo constituye el desarrollo de un sistema de gerencia e inspección de obras automatizada, la idea es que una sola persona pueda abarcar el mayor número de inspecciones de obras utilizando la herramienta tecnológica que se ha diseñado.

El diseño de estos programas implica la incorporación de personal preparado en el área de informática y el uso de las herramientas más sofisticadas para el desarrollo de software, para ello se busca la participación de estudiantes en calidad de tesistas; esto trae varias ventajas: disminuye la inversión, ya que sólo se le paga el tiempo dedicado al desarrollo de los programas; la calidad del trabajo es excelente, por la motivación que el estudiante siente dada la utilidad de este tra- bajo de grado para la culminación de su carrera; hay una sustentación teórica importante, aportada por el tutor académico quien es especialista en el área de informática; el procedimiento seguido es sistemático, ya que es obligatorio en un trabajo de investigación $y$, por último, el programa se crea en correspondencia con las necesidades de la empresa, ya que el tutor empresarial de la tesis es el Gerente de I\&D.

\section{Conclusiones}

La Ai es una técnica válida para cambiar el rumbo de una organización hacia el logro de sus metas estratégicas y hacia la obtención de resultados a corto plazo. Con la aplicación de la Ri en $\mathrm{PROINCl}$ se han logrado avances decisivos en medidas importantes de rendimiento, reflejados estos avances no sólo en la automatización, en la reducción del tamaño de la empresa, en la reorganización, sino también en la calidad, costos, rapidez y satisfacción del cliente. El esfuerzo de reingeniería llevado a cabo permite hoy afirmar que ésta es una empresa competitiva ya que tiene la cualidad de la competitividad frente al resto de las firmas dentro de su área de competencia.

El redimensionamiento llevado a cabo se refleja en el diseño de una nueva estructura organizativa la cual reporta una serie de ventajas y propicia la sinergia necesaria para optimizar los flujos de trabajo, entre esas ventajas están las siguientes:

* Las personas claves para un proyecto trabajan en equipo. A cada proyecto se asigna únicamente el número de 
Reingeniería y competitividad

Brígida Ferrer, Rixia Villalobos y Eudio Omar Barboza

personas que necesita, evitándose así las duplicaciones innecesarias y la segmentación del trabajo, aunado al hecho de que disminuyen los costos operativos por la racionalización de los recursos.

* El equipo de proyectos funciona dentro de la propia estructura organizacional propuesta y se nutre de las unidades operativas mencionadas de Desarrollo, Técnica y de Obras. Estas unidades operativas le dan apoyo al proyecto en términos de recursos técnicos, personal y procedimientos. Bajo esta concepción se rompe la estructura piramidal y el proyecto es sometido a la discusión del equipo que cuenta con la dirección del líder.

* Esta estructura matricial exige el desarrollo de destrezas extraordinarias en cuanto a las relaciones interpersonales intensas que se ponen en juego entre los miembros del equipo; esta situación es un elemento motivador para estimular el desarrollo de tales destrezas a la luz de los nuevos procesos de reingeniería.

Analizar los procesos básicos del negocio bajo el enfoque de la reingenieria permitió descubrir que la "ejecución del trabajo" es bastante costosa para la empresa dado el alto grado de especialización que requiere, por lo tanto, es necesario enfatizar en otras actividades más rentables y menos problemáticas de la misma área de ingeniería como lo son las inspecciones de obras y las promociones de viviendas.

A partir del rediseño se internalizó el poder que otorga la generación de tecnología en la empresa y se entendió que los profesionales altamente especializados, costosos y escasos, no son necesarios como personal fijo; su situación puede ser cambiada por la figura de empleados bien remunerados que prestan un servicio cuando se les requiera. El tipo de empleados fijos deben ser aquellos del área de computación o de sistemas que vayan creando las herramientas automatizadas que multipliquen la capacidad de trabajo de la empresa y reflejen su competitividad.

La Ri permite cambiar los procesos técnicos básicos del negocio pero también genera beneficios al recurso humano de la organización ya que induce la capacitación, educación y desarrollo del personal como herramienta obligatoria para el éxito del proyecto de Ri. En este sentido no sólo se les debe proveer de las destrezas necesarias para participar como empleado multioficio sino que además deben ser preparados para encarar sanamente y resolver los conflictos intrapersonales que pudiera generar la incertidumbre propia de una nueva situación, su aceptación o resistencia.

En el caso estudiado el diseño técnico se cumplio favorablemente con importantes resultados para la organización, sin embargo, el desarrollo del recurso humano con miras a su incorporación integral a los nuevos procesos no se desenvolvió simultáneamente al diseño técnico lo cual amenaza la visión holística del proyecto de $\mathbf{R i}$.

La aplicación de esta técnica permitió a los investigadores involucrados probar una vez mas que ninguna técnica gerencial diseñada fuera de nuestro contexto puede aplicarse a las organizaciones empresariales venezolanas sin realizar las adaptaciones necesarias a cada caso particular; el entorno socio-econó- 
mico cambiante y las particularidades de cada sector así lo exigen para intentar el éxito.

\section{Referencias bibliográficas}

Alvarez H., Galindez Isabel y Romero Lorena (1996). Diseño de Planos de Planta de Estructuras de Concreto Armado en Ambiente AutoCAD. Tesis de Grado. Universidad del Zulia. Facultad de Ingeniería. Escuela de Ingeniería Civil. Maracaibo, Venezuela.

Hammer, Michael y Champy, James. (1994)
Reingeniería. Grupo Editorial Norma Bogotá, Colombia.

Morris, Daniel y Brandon, Joel. (1994) Reingeniería. Cómo aplicarla con éxito en los negocios. Mc. Graw-Hill. Bogotá, Colombia.

Manganelli, Raymond y Klein, Mark, (1995) Cómo hacer Reingenieria. Grupo Editorial Norma. Bogotá, Colombia,

Porter, Michael. (1985) Estrategia Competitiva. Compañía Editorial Continental S.A. (C.E.C.S.A.). México.

Senge, Peter. (1990) La Quinta Disciplina: el arte y la práctica de la organización abierta al aprendizaje. Ediciones Juan Granica. España. 